\title{
Enzyme replacement therapy desensitization in a child with infantile onset Pompe disease
}

\author{
Theresa Shu Wen Toh, ${ }^{1}$ Kok Wee Chong, ${ }^{2}$ Anne Eng Neo Goh, ${ }^{2}$ Jasmine Chew Yin Goh, ${ }^{4}$ Teck Wah Ting, ${ }^{3}$ Ee Shien Tan, ${ }^{3}$ Si Hui Goh ${ }^{2}$
}

\section{Abstract}

Background: Enzyme replacement therapy significantly reduces morbidity and mortality in patients with Pompe disease. Development of hypersensitivity reactions to enzyme replacement therapy is common and can adversely affect disease outcomes when treatment is halted or delayed.

Objective: Our institution reports a case of successful alglucosidase alfa enzyme replacement therapy desensitisation in a 9-year-old girl with infantile onset Pompe disease.

Methods: A desensitisation protocol was tailored to our patient with the help of a multidisciplinary team including the allergist, geneticist, nurses and pharmacists.

Results: For our patient, desensitisation was successful using a multi-step three-fold dose escalation protocol.

Conclusion: Desensitisation is possible in individuals with hypersensitivity reactions to enzyme replacement. Desensitisation protocols need to be tailored according to the patient's needs and responses to find a protocol that is safe, effective and simple.

Key words: Pompe disease, alglucosidase alfa, Enzyme replacement, Desensitisation, Hypersensitivity

\section{Citation:}

Toh, T. S. W., Chong, K. W., Goh, A. E. N., Goh, J. C. Y., Ting, T. W., Tan, E. S., Goh, S. H. (2022). Enzyme replacement therapy desensitization in a child with infantile onset Pompe disease. Asian Pac J Allergy Immunol, 40(4), 414-417. https://doi.org/10.12932/ap-060919-0638

\section{Affiliations:}

${ }^{1}$ Department of Paediatric Medicine,

KK Women's and Children's Hospital, Singapore

2 Allergy Service, Department of Paediatric Medicine,

KK Women's and Children's Hospital, Singapore.

3 Genetic Service, Department of Paediatric Medicine,

KK Women's and Children's Hospital, Singapore

${ }^{4}$ Division of Nursing, Nursing Specialist Services,

KK Women's and Children's Hospital, Singapore

Corresponding author:

Goh Si Hui

KK Women's and Children's Hospital,

100 Bukit Timah Road, Singapore 229899

E-mail: goh.si.hui@singhealth.com.sg

\section{Introduction}

Pompe disease (PD) is a rare inherited disorder caused by mutations in acid-alpha-glucosidase (GAA) gene that codes for the enzyme alpha-glucosidase. Alpha-glucosidase is responsible for breakdown of glycogen into glucose for energy use. Loss of function of this enzyme results in accumulation of glycogen predominantly in skeletal muscle fibres. There is a wide clinical spectrum of this disease ranging from lethal infantile onset form to progressive late onset form characterized by varying degree of muscle weakness, respiratory insufficiency and eventual death usually from respiratory failure. ${ }^{1}$ Incidence of Pompe disease varies amongst different ethnicities with a reported incidence amongst Chinese population of 1 in 50,000. ${ }^{2}$

Enzyme replacement therapy (ERT) has been proven to reduce morbidity and mortality in $\mathrm{PD} .^{3}$ The first two ERT approved as orphan drugs by the Food and Drug Administration (FDA) were Myozyme and Lumizyme. ${ }^{4}$ Myozyme was indicated for use in infantile onset PD since 2006. Lumizyme was initially indicated for use in patients 8 years and older in 2010 and extended for use in infantile onset PD in 2014. The main boxed warning for both drugs is the development of infusion associated reaction (IAR), 
including hypersensitivity reactions. Post-marketing trials report an estimated incidence of hypersensitivity reactions in $10 \%$ of patients and anaphylaxis in less than $1 \%{ }^{5}$ Strategies to deal with IARs include slowing the infusion rate, prolonging each infusion rate ramp step, temporarily halting the infusion and pre-medicating with steroids or antihistamines. However, if these measures fail, desensitisation is strongly indicated.

\section{Report of Case}

This is a 9-year-old Chinese girl born to nonconsanguineous parents. She presented at 7 months with hypotonia, delayed motor milestones and cardiac failure secondary to hypertrophic cardiomyopathy. Investigations revealed deficient GAA activity in both skin fibroblast cells (1.06 nmol/hr/mg protein; control reference $260 \mathrm{nmol} / \mathrm{hr} /$ mg protein) and blood spot (3.9 pmol/punch/hour; control reference 10.0-49.0 $\mathrm{pmol} / \mathrm{punch} / \mathrm{hour})$. DNA sequencing for GAA gene was also done that confirmed presence of a homozygous GAA mutation involving single nucleotide change (c.1935C>A) in exon 14 which is a common mutation seen in the Chinese population. ${ }^{6}$ This assay used polymerase chain reaction amplification followed by Sanger DNA sequencing to detect mutations in the GAA gene that cause Pompe disease. Cross-reactive immunologic material (CRIM) status was positive in our patient and was determined via Western Blot analyses on cell lysates derived from skin fibroblast cells. GAA protein bands were identified using a pool of polyclonal antibodies made against placental GAA protein that recognised both processed and unprocessed forms of GAA protein. Her other comorbidities include restrictive lung disease requiring nocturnal bi-level positive pressure ventilation and gastro-oesophageal reflux disease for which a gastrostomy creation and fundoplication was done. She is at present wheelchair-bound and fully assisted in her activities of daily living.
She was treated with Myozyme infusion at $20 \mathrm{mg} / \mathrm{kg}$ commencing at 9 months of age. In September 2016, 6 years after initiation of ERT, she developed hypersensitivity reactions. She was noticed to have an urticarial rash over the neck and forehead within two hours onset from infusion administration but otherwise no hemodynamic or respiratory compromise. For the subsequent Myozyme infusions, she was pre-medicated with cetirizine, prednisolone and the infusion was slowed to a third of the initial rate. In spite of the instituted measures, she developed significant and persistent urticaria. She was unable to complete her full dose of Myozyme for four cycles.

Desensitisation was proposed after a multi-disciplinary discussion involving genetics-metabolic team, allergy team and pharmacists. Her parents gave informed consent. Skin prick test was negative, with histamine control 8.5 $\mathrm{mm}$, negative control $0 \mathrm{~mm}$, Myozyme (neat, $5 \mathrm{mg} / \mathrm{ml}$ ) 2 $\mathrm{mm}$. She was negative for recombinant alpha-glucosidase (rhGAA) IgG antibodies as determined by enzyme-linked immunosorbent assay (ELISA, Genzyme Laboratory). We did not perform rh-GAA IgE antibodies and intradermal skin test. She was commenced on the first desensitisation protocol adapted from El-Gharbawy et al's report published in $2011 .^{7}$ Her total dose was dropped to $10 \mathrm{mg} / \mathrm{kg}$ weekly and administered via the protocol shown in Table 1, with tepwise 10-fold dose increments. However, she continued to develop significant urticarial rashes at point of escalation to $500 \mathrm{mcg} / \mathrm{kg} / \mathrm{hr}$ of Myozyme infusion rate. The patient was clinically stable, however there was generalized and distressing urticarial rash which progressed despite administration of repeated doses oral non-sedative antihistamine (cetirizine). Thus, only $1.5 \mathrm{mg} / \mathrm{kg}$ (15\%) of the intended dose was completed.

Table 1.

\begin{tabular}{|c|c|c|c|c|c|c|c|}
\hline Step & $\begin{array}{c}\text { Concentration } \\
(\mathbf{m c g} / \mathbf{m l})\end{array}$ & $\begin{array}{c}\text { Infusion rate } \\
(\mathbf{m c g} / \mathbf{k g} / \mathbf{h r})\end{array}$ & $\begin{array}{c}\text { Time } \\
(\mathbf{m i n})\end{array}$ & $\begin{array}{c}\text { Volume infused } \\
(\mathbf{m l})\end{array}$ & $\begin{array}{c}\text { Dose administered } \\
(\mathbf{m c g})\end{array}$ & $\begin{array}{c}\text { Cumulative dose } \\
(\mathbf{m c g})\end{array}$ & $\begin{array}{c}\text { Fold increase } \\
\text { per step }\end{array}$ \\
\hline 1 & 0.002 & 0.002 & 30 & 10 & 0.02 & 0.02 & 0 \\
\hline 2 & 0.02 & 0.018 & 30 & 9 & 0.18 & 0.2 & 9 \\
\hline 3 & 0.2 & 0.18 & 30 & 9 & 1.8 & 2 & 10 \\
\hline 4 & 2 & 1.8 & 30 & 9 & 18 & 20 & 10 \\
\hline 5 & 20 & 18 & 30 & 9 & 180 & 200 & 10 \\
\hline 6 & 200 & 180 & 30 & 9 & 1,800 & 2,000 & 10 \\
\hline $7^{*}$ & 2,000 & 500 & 30 & 2.5 & 5,000 & 7,000 & 2.7 \\
8 & 2,000 & 1,000 & 30 & 5 & 10,000 & 17,000 & 2 \\
9 & 2,000 & 2,000 & 30 & 10 & 20,000 & 37,000 & 2 \\
10 & 2,000 & 4,000 & 70 & 81 & 162,000 & 199,000 & 2 \\
\hline
\end{tabular}

Protocol details: Total dose $10 \mathrm{mg} / \mathrm{kg}$, Weight $20 \mathrm{~kg}$, Total dose $200 \mathrm{mg}$, Total time $=366 \mathrm{~min}\left(6.6\right.$ hours) (adapted from Gharbawy et al 2011) ${ }^{6}$ ${ }^{*}$ Persistent urticarial rash noted at $500 \mathrm{mcg} / \mathrm{kg} / \mathrm{hr}$ with inability to proceed beyond this rate (shaded rows were not carried out in our patient) 
Table 2.

\begin{tabular}{|c|c|c|c|c|c|c|c|}
\hline Step & $\begin{array}{l}\text { Concentration } \\
(\mathbf{m c g} / \mathrm{ml})\end{array}$ & $\begin{array}{c}\text { Infusion rate } \\
(\mathrm{mcg} / \mathrm{kg} / \mathrm{hr})\end{array}$ & $\begin{array}{l}\text { Time } \\
(\mathrm{min})\end{array}$ & $\begin{array}{l}\text { Volume infused } \\
(\mathrm{ml})\end{array}$ & $\begin{array}{l}\text { Dose administered } \\
\text { (mcg) }\end{array}$ & $\begin{array}{l}\text { Cumulative dose } \\
\text { (mcg) }\end{array}$ & $\begin{array}{c}\text { Fold increase } \\
\text { per step }\end{array}$ \\
\hline 1 & 0.01 & 0.01 & 30 & 10 & 0.1 & 0.1 & 0 \\
\hline 2 & 0.1 & 0.025 & 30 & 2.5 & 0.25 & 0.35 & 2.5 \\
\hline 3 & 0.1 & 0.065 & 30 & 6.5 & 0.65 & 1 & 2.6 \\
\hline 4 & 1 & 0.25 & 30 & 2.5 & 2.5 & 3.5 & 3.8 \\
\hline 5 & 1 & 0.65 & 30 & 6.5 & 6.5 & 10 & 2.6 \\
\hline 6 & 10 & 2.5 & 30 & 2.5 & 25 & 35 & 3.8 \\
\hline 7 & 10 & 6.5 & 30 & 6.5 & 65 & 100 & 2.6 \\
\hline 8 & 100 & 25 & 30 & 2.5 & 250 & 350 & 3.8 \\
\hline 9 & 100 & 65 & 30 & 6.5 & 650 & 1,000 & 2.6 \\
\hline 10 & 1,000 & 250 & 30 & 2.5 & 2,500 & 3,500 & 3.8 \\
\hline 11 & 1,000 & 375 & 30 & 3.75 & 3,750 & 7,250 & 1.5 \\
\hline $12^{*}$ & 1,000 & 500 & 30 & 5 & 5,000 & 12,250 & 1.3 \\
\hline 13 & 1,000 & 750 & 30 & 7.5 & 7,500 & 19,750 & 1.5 \\
\hline 14 & 1,000 & 1,000 & 30 & 10 & 10,000 & 29,750 & 1.3 \\
\hline 15 & 1,000 & 1,500 & 30 & 15 & 15,000 & 44,750 & 1.5 \\
\hline 16 & 1,000 & 2,000 & 82 & 55.25 & 55,250 & 100,000 & 1.3 \\
\hline 17 & 1,000 & 3,000 & 60 & 60 & 60,000 & 160,000 & 1.5 \\
\hline 18 & 1,000 & 4,000 & 30 & 40 & 40,000 & 200,000 & 1.3 \\
\hline
\end{tabular}

Protocol details: Dose $10 \mathrm{mg} / \mathrm{kg}$, Weight $20 \mathrm{~kg}$, Total dose $200 \mathrm{mg}$, Total time $=622 \mathrm{~min}(10.3 \text { hours) (adapted from Karagol et al } 2012)^{7}$ *Occasional mild urticarial rashes requiring prolongation of infusion time

From the first desensitisation attempt, pertinent learning points were firstly the crucial role of pharmacist involvement to prepare Myozyme microdilution at timely intervals as the diluted solutions can only last for 24 hours. Pre-diluting the whole batch beforehand resulted in wastage of an expensive drug when protocol duration was prolonged secondary to urticarial reactions. Secondly, our patient fared better with conversion to a gentle 3-fold concentration increment, adopted from a case description by Karagol et al. ${ }^{8}$ For the second infusion using the new protocol and pre-medication of oral prednisolone, she experienced reactions similarly at $500 \mathrm{mcg} / \mathrm{kg} / \mathrm{hr}$ requiring a decrease in infusion rate to 250 $\mathrm{mcg} / \mathrm{kg} / \mathrm{hr}$ and gradual increase up to $2000 \mathrm{mcg} / \mathrm{kg} / \mathrm{hr}$ (2 $\mathrm{mg} / \mathrm{kg} / \mathrm{hr}$ ) with rashes noted at each two-fold concentration increment. Despite reactions, she was able to complete the full Myozyme dose on the second cycle. After modification and tailoring for the patient's response, we present the final steps of our desensitisation protocol in Table 2. After 7 infusion cycles, we were able to stop pre-medications and after 9 infusion cycles, we were able to successfully convert the patient back to full dose of $20 \mathrm{mg} / \mathrm{kg}$ Myozyme and compress increment steps for shorter infusion duration. Three years on from initial desensitisation, she is tolerating her 2-weekly Myozyme infusions well.

\section{Discussion}

IAR to Myozyme are frequent in patients with Pompe disease. ${ }^{3}$ A Phase III trial of Myozyme in infantile onset Pompe disease highlighted that 11 of the 18 patients experienced 164 IARs of mild to moderate severity including rash, fever, urticaria and desaturation. ${ }^{9}$ IARs were typically managed by slowing or interrupting infusions. However, adjustment of infusion rate and administration of pre-medications were ineffective in our patient.

The immunologic mechanisms behind IARs are not well understood. Postulations include IgE mediated mechanisms ${ }^{10}$ and non IgE mediated mechanisms of IgG antibodies with complement activation, ${ }^{7}$ cytokine release, ${ }^{11}$ and direct mast cell stimulation. ${ }^{5}$ As IARs of various mechanisms have similar presentations, clinicians are unable to use clinical history to predict and guide management. In case reports of IgE mediated IAR, skin prick test was negative in all patients and $\operatorname{IgE}$ sensitisation was proven by anti-rhGAA $\operatorname{IgE}$ and intradermal testing of varying concentration from $1: 1000^{8}$ to $1: 100{ }^{10,12}$ For our patient, an IgE mediated mechanism was highly likely given her negative anti-rhGAA IgG and a skin prick test that measured $2 \mathrm{~mm}$ above negative diluent. After discussion with her parents, factoring in potential discomfort with intradermal testing and health resources, we proceeded with desensitisation therapy without pursuing the cause of IAR mechanism. 
As recommended in the 2010 European Academy of Allergy and Clinical Immunology (EAACI) consensus statement on rapid desensitisation for drug hypersensitivity, ${ }^{13}$ when the drug involved is essential for optimal therapy, desensitisation may be performed. Desensitisation is the induction of a temporary state of tolerance by introducing the culprit drug in increasing subtherapeutic doses. Desensitisation is performed only in IgE independent or IgE dependent reaction in which mast cell degranulation occurs. ${ }^{14}$ Whilst desensitisation to Myozyme has been reported in literature, the numbers are small and mostly published as case reports ${ }^{11}$ or case series ${ }^{12}$ with less than 10 patients per published protocol. The first protocol we used from Gharbawy was a simple 10-step, 10-fold escalation protocol with clearly stipulated rescue medications and safety guidelines. Unfortunately for our patient, the concentration escalation was too rapid and she continued to have distressing reactions prompting a change to the Karagol protocol. The main difference between our protocol and the original Karagol protocol was the more gradual stepwise dose escalation from step 13 onwards. Whilst it is common to get breakthrough reactions at the first course of desensitisation, our team opted to modify desensitisation protocol based on individual patient response.

One of the key predictors of ERT tolerance is the crossreactive immunologic material (CRIM) status of the patient. Patients with detectable endogenous GAA are CRIM positive and tend to tolerate ERT well. In contrast, patients without detectable endogenous GAA are CRIM negative, develop high and sustained IgG antibody titres to exogenous recombinant GAA (rhGAA), and associated with poor tolerance and efficacy of ERT resulting in poor clinical outcomes. In patients who are CRIM negative, immunomodulation is an option in IgG antibody naïve patients as prophylaxis and patients with high antibody titres as treatment. Modalities of immunomodulation include rituximab, methotrexate and intravenous immunoglobulins. ${ }^{15,16}$ As our patient was CRIM positive and anti-rhGAA IgG negative, immunomodulation was not an option.

In conclusion, it is possible to safely desensitise PD patients with ERT hypersensitivity for continuation of outcome modifying treatment. Desensitisation involves the multidisciplinary cooperation of nurses, pharmacists and doctors in the allergy, genetics and inpatient-monitoring team. Desensitisation protocols need to be tailored according to the patient's needs and responses to find a protocol that is safe, effective and simple.

\section{Acknowledgement}

Pharmacists - Praveena Kandasamy, Kelvin Xu Shaorong, Ashley Lim Shiyuan

\section{References}

1. Hagemans ML, Winkel LP, Van Doorn PA, Hop WJ, Loonen MC, Reuser AJ, et al. Clinical manifestation and natural course of late-onset Pompe's disease in 54 Dutch patients. Brain. 2005;128(Pt 3):671-7.

2. Kishnani PS, Hwu WL, Mandel H, Nicolino M, Yong F, Corzo D, et al. A retrospective, multinational, multicenter study on the natural history of infantile-onset Pompe disease. J Pediatr. 2006;148(5):671-6.

3. Nicolino M, Byrne B, Wraith JE, Leslie N, Mandel H, Freyer DR, et al. Clinical outcomes after long-term treatment with alglucosidase alfa in infants and children with advanced Pompe disease. Genet Med. 2009;11(3):210-9.

4. Guo J, Kelton CM, Guo JJ. Recent developments, utilization, and spending trends for pompe disease therapies. Am Health Drug Benefits. 2012;5(3):182-9.

5. Israel MoH. [Internet]. Jerusalem: Ministry of Health Israel; c2019. Myozyme information leaflet [cited 2019 Aug 1]; [2 p.]. Available from: https://wwwoldhealthgovil/units/pharmacy/trufot/alonim/ Myozyme_dr_1403788015997pdf.

6. Wan L, Lee CC, Hsu CM, Hwu WL, Yang CC, Tsai $\mathrm{CH}$, et al. Identification of eight novel mutations of the acid alpha-glucosidase gene causing the infantile or juvenile form of glycogen storage disease type II. J Neurol. 2008;255(6):831-8.

7. El-Gharbawy AH, Mackey J, DeArmey S, Westby G, Grinnell SG, Malovrh P, et al. An individually, modified approach to desensitize infants and young children with Pompe disease, and significant reactions to alglucosidase alfa infusions. Mol Genet Metab. 2011;104(1-2):118-22.

8. Karagol IH, Bakirtas A, Yilmaz O, Topal E, Kucukcongar A, Ezgu FS, et al. Desensitisation of the youngest patient with Pompe disease in response to alglucosidase alfa. Allergol Immunopathol (Madr). 2014;42(4):372-5.

9. Kishnani PS, Corzo D, Nicolino M, Byrne B, Mandel H, Hwu WL, et al. Recombinant human acid [alpha]-glucosidase: major clinical benefits in infantile-onset Pompe disease. Neurology. 2007;68(2):99-109.

10. Lipinski SE, Lipinski MJ, Burnette A, Platts-Mills TA, Wilson WG. Desensitization of an adult patient with Pompe disease and a history of anaphylaxis to alglucosidase alfa. Mol Genet Metab. 2009;98(3):319-21.

11. Baruteau J, Broomfield A, Crook V, Finnegan N, Harvey K, Burke $D$, et al. Successful Desensitisation in a Patient with CRIM-Positive Infantile-Onset Pompe Disease. JIMD Rep. 2014;12:99-102.

12. Capanoglu M, Dibek Misirlioglu E, Azkur D, Vezir E, Guvenir H, Gunduz M, et al. IgE-Mediated Hypersensitivity and Desensitisation with Recombinant Enzymes in Pompe Disease and Type I and Type VI Mucopolysaccharidosis. Int Arch Allergy Immunol. 2016;169(3):198-202.

13. Cernadas JR, Brockow K, Romano A, Aberer W, Torres MJ, Bircher A, et al. General considerations on rapid desensitization for drug hypersensitivity - a consensus statement. Allergy. 2010;65(11):1357-66.

14. de Las Vecillas Sanchez L, Alenazy LA, Garcia-Neuer M, Castells MC. Drug Hypersensitivity and Desensitizations: Mechanisms and New Approaches. Int J Mol Sci. 2017;18(6).

15. Doerfler PA, Nayak S, Corti M, Morel L, Herzog RW, Byrne BJ. Targeted approaches to induce immune tolerance for Pompe disease therapy. $\mathrm{Mol}$ Ther Methods Clin Dev. 2016;3:15053.

16. Messinger YH, Mendelsohn NJ, Rhead W, Dimmock D, Hershkovitz E, Champion $\mathrm{M}$, et al. Successful immune tolerance induction to enzyme replacement therapy in CRIM-negative infantile Pompe disease. Genet Med. 2012;14(1):135-42. 